\title{
ESTOQUE DE CARBONO NA BIOMASSA DE PLANTAÇÕES DE EUCALIPTO NA REGIÃO CENTRO-LESTE DO ESTADO DE MINAS GERAIS ${ }^{1}$
}

Alcides Gatto², Nairam Félix de Barros ${ }^{3}$, Roberto Ferreira de Novais ${ }^{3}$, Ivo Ribeiro da Silva ${ }^{3}$, Hélio Garcia Leite ${ }^{4}$ e Ecila Mercês de Albuquerque Villani ${ }^{5}$

\begin{abstract}
RESUMO - Os ecossistemas florestais representam alternativa viável para mitigar o aumento da concentração de $\mathrm{CO}_{2}$ na atmosfera, via fixação do carbono (C) pelas árvores e seu armazenamento na biomassa e no solo, mas tais informações são escassas. Este trabalho teve como objetivo avaliar a produção de matéria seca da parte aérea, de raízes e da manta orgânica depositada sobre o solo e o estoque de C de árvores em plantações de eucalipto com diferentes idades na região Centro-Leste do Estado de Minas Gerais, abrangendo cinco regiões: Cocais (CO), Rio Doce (RD), Sabinópolis (SA), Santa Bárbara (SB) e Virginópolis (VI). Quanto à produção de matéria seca, a região mais produtiva, aos 84 meses de idade, foi SA, com 32,80 t ha-1 ano ${ }^{-1}$ de parte aérea e raízes, decrescendo nos anos seguintes até atingir 31,18 t ha-1 ano-1 aos 120 meses de idade. Para essa mesma comparação, nas regiões de RD e SB observaram-se produtividades de 29,92 e 29,70 tha-1 ano-1 aos 84 meses e 21,09 e 25,21 tha-1 ano ${ }^{-1}$ aos 120 meses de idade, respectivamente. Constatou-se que a estabilização da produtividade ocorreu após 96 meses de idade em SA e aos 84 meses para as regiões de RD e SB. No tocante às regiões de $\mathrm{CO}$ e VI, a produtividade e o estoque de $\mathrm{C}$ médio anuais mantiveram taxas crescentes até 120 meses de idade, indicando que a maior produtividade ocorre em idades mais avançadas. A produtividade e o estoque de $\mathrm{C}$ médio dessas plantações foram, respectivamente, de 26,96 t ha $\mathrm{ano}^{-1}$ de biomassa e 13,64 t ha ${ }^{-1}$ ano $^{-1}$ de C.
\end{abstract}

Palavras-chave: Componentes da árvore, Equações alométricas e Sequestro de carbono.

\section{CARBON STOCK IN THE BIOMASS OF EUCALYPTUS CROPS IN CENTRAL- EAST REGION OF THE STATE OF MINAS GERAIS - BRAZIL}

\begin{abstract}
Forest ecosystems are a viable possibility to mitigate the increase in concentration of $\mathrm{CO}_{2}$ in the atmosphere by carbon (C) sequestration by the trees and its storage in biomass and in the soil, but such information are scarce. The main objective of this study was to assess production of dry matter of aerial section, roots and litter layer on the soil and C stock of trees in eucalyptus crops in the Central-East region of Minas Gerais State, Brazil, in five regions: Cocais (CO), Rio Doce (RD), Sabinópolis (SB), Santa Bárbara (SA), and Virginópolis (VI). Regarding dry matter production, the most productive region was $S A$, with $32.80 \mathrm{tha}^{-1} \mathrm{yr}^{-1}$, in trees at 84 months of age and 21.09 and $25.21 \mathrm{tha}^{-1} \mathrm{yr}^{-1}$ in trees at 120 months of age, respectively. Stabilization of productivity was after 96 months of age in $S A$ and at 84 months of age for RD and SB. For region CO and VI, productivy and average year C stock maintained increasing rates until 120 months of age, showing that the greatest productivy occurs in advanced ages. Productivity and average C stock of those crops were $26.96 \mathrm{tha^{-1 }} \mathrm{yr}^{-1}$ of biomass and $13.64 t \mathrm{ha}^{-1} \mathrm{yr}^{-1} \mathrm{C}$.
\end{abstract}

Keywords:Allometric equations, Carbon sequestration and Tree components

\footnotetext{
${ }^{1}$ Recebido em 24.09.2008 e aceito para publicação em 20.04.2011.

${ }^{2}$ Departamento de Engenharia Florestal, Universidade de Brasília, UnB, Brasil. E-mail: <alcidesgatto@unb.br>.

${ }^{3}$ Departamento de Solos, Universidade Federal de Viçosa, UFV, Brasil. E-mails: <nfbarros@ufv.br>, <rfnovais@ufv.br>e <ivosilva@ufv.br>.

${ }^{4}$ Departamento de Engenharia Florestal, UFV. E-mail: <hgleite@ufv.br>.

${ }^{5}$ Pós Doutoranda do Departamento de Solos, bolsista da FAPEMIG, UFV. E-mail: <ecilavillani@hotmail.com>.
} 


\section{INTRODUÇÃO}

O setor florestal desponta como uma das alternativas viáveis para mitigar o aumento da concentração de $\mathrm{CO}_{2}$ na atmosfera, via fixação do carbono (C) pelas plantas arbóreas, principalmente. No Brasil, o clima tropical predominante e a grande extensão territorial contribuem para que as plantações florestais apresentem altas taxas de crescimento vegetativo, acrescidas, ainda, da disponibilidade de mão de obra e técnicas inovadoras (STAPE et al., 2008).

As espécies florestais distinguem-se das demais espécies vegetais por possuírem a capacidade de fixar C por anos ou décadas e armazená-lo na forma de madeira (LITTON et al., 2007). A quantidade de C fixado e o seu tempo de residência no ecossistema dependem, entre outros fatores, da idade da planta, do componente onde o C é alocado e do uso destinado à madeira (DIAZBALTEIRO; RODRIGUEZ, 2006).

Constatou-se em plantações de Eucalyptus grandis, em dois sítios de Cerrado, que a produção de biomassa dos componentes das árvores variou com a idade do povoamento e com a capacidade produtiva do sítio. No sítio de maior capacidade produtiva, em Bom Despacho, MG as raízes contribuíram com $14 \%$ do total de $\mathrm{C}$ armazenado nas árvores, e, naquele de menor capacidade (Carbonita, MG), essa contribuição chegou a 30\% do C total das árvores. Verificou-se que o percentual de biomassa correspondente às folhas e aos galhos diminuiu em idades mais avançadas (12,7\%) do que em idades mais jovens (32,3\%), enquanto a biomassa da manta orgânica no solo aumentou até os 52 meses de idade (FERREIRA, 1984). Stape et al. (2008) verificaram que as folhas de E. grandis $x$ urophylla armazenaram $8,8 \%$ do $C$ total das árvores, os galhos 7,8\% e o lenho 83,4\% em plantios localizados no Sul da Bahia.

Com relação à conversão de biomassa em matéria seca, em C, 0,50 é o fator comumente empregado para essa conversão (IPCC, 1996). Gifford (2000) determinou o conteúdo de $\mathrm{C}$ nos tecidos e na serapilheira de 19 espécies de árvores nativas da Austrália, 15 das quais eram eucalipto, reportando que o conteúdo médio de C não passava de 50,3\%, sugerindo um fator de conversão de $50 \pm 2 \%$ para abranger as diversas espécies estudadas.

O total de C em uma árvore de eucalipto, em média, apresenta a seguinte distribuição: tronco 65\%, copa $13 \%$ e raízes $22 \%$ (BRASIL, 2001). Pode-se observar que o tronco representa mais da metade da biomassa de uma plantação de eucalipto. Assim, ao desprezar a copa e as raízes para cálculo do C, subestima-se o real valor de $\mathrm{C}$ estocado. Contudo, devido à dificuldade de obtenção e aferição do estoque de C nesses últimos componentes, muitas vezes esses são negligenciados na predição do estoque total de C. Em decorrência da dificuldade de medição e da necessidade de redução de tempo e recursos destinados à obtenção da biomassa de galhos e folhas, esses componentes podem ser estimados como proporção da biomassa do tronco (LOZA-BALBUENA, 2001). Paixão et al. (2006) estimaram a produção de biomassa radicular em 21\% da biomassa total em plantios de E. grandis com 6 anos de idade.

Para estimar a biomassa e quantificar o estoque de C orgânico em plantações florestais, há métodos que vão desde a análise destrutiva com o abate das árvores (métodos diretos) e não destrutiva (métodos indiretos) até a estimativa por processamento digital de imagens por satélite. Em geral, os métodos de estimativa do estoque de C orgânico fazem uso de modelos de regressão cujas variáveis independentes são a biomassa total e o fator de conversão $(0,50)$, pressupondo-se que $50 \%$ da biomassa é constituída por C (1,0 g de matéria seca corresponde $=0,5$ g de C orgânico ou 1,7 g de $\mathrm{CO}_{2}$ ) (BROWN et al., 1986).

O modelo desenvolvido por Dewar e Cannell (1992) descreve como o depósito e o fluxo deC ocorrem em plantações florestais equiâneas, periodicamente colhidas e replantadas. Outros modelos levam em consideração a vida útil do produto madeireiro e o período de rotação para máxima produção de biomassa da floresta (LELES et al., 1994). Além desses dois, devem ser considerados os desenvolvidos por Schroeder (1992), Alvarez (1993), Flinte Richards (1994), Hoene Solberg (1994), Delaney et al. (1997), Ortiz (1997), Bashkin e Binkley (1998) e Vélez e Arango (2001) e, recentemente, Montagu et al. (2005) e Sales et al. (2006).

Uma abordagem prática, porém nem sempre exequível e muitas vezes onerosa, para estimar o estoque de C orgânico em plantações florestais é baseada no volume convertido em biomassa seca, para posterior obtenção do estoque (BEETS et al., 1999). Inicialmente, é necessário obter o volume e, ou, a biomassa seca dos componentes aéreos e subterrâneos. O componente aéreo é 
representado pelo tronco (lenho+casca) e pela copa (folhas+galhos), e o subterrâneo, pelo sistema radicular (raízes grossas, finas e pivotante). Posteriormente, estima-se o estoque de C orgânico por meio do produto da biomassa seca pelo teor de $\mathrm{C}$ de cada componente para estimativas mais exatas, ou com um fator único, 0,50, tradicionalmente utilizado para essa conversão, obtendo, contudo, estimativas menos exatas. Considera-se que o estoque total de C orgânico na biomassa é a soma do estocado na biomassa dos componentes das árvores mais o estocado na manta orgânica.

Nesse contexto, este trabalho teve como objetivo avaliar o estoque de $\mathrm{C}$ na biomassa de plantações de eucalipto na região Centro-Leste do Estado de Minas Gerais.

\section{MATERIAL E MÉTODOS}

O estudo abrangeu cinco regiões distintas quanto às características edafoclimáticas, situadas na região Centro-Leste de Minas Gerais, a saber: Cocais (CO), Rio Doce (RD), Sabinópolis (SA), Santa Bárbara (SB) e Virginópolis (VI), localizadas entre as coordenadas geográficas de 18³8’ a 1959' (latitude Sul) e $42^{\circ} 25^{\prime}$ a $43^{\circ} 20^{\prime}$ (longitude Oeste), de Greenwhich.

O clima predominante nas regiões de SB, VI e SA, pela classificação de Köppen, é do tipo Cwa, temperado chuvoso-mesotérmico, em que a temperatura média do mês mais frio é inferior a 18 ${ }^{\circ} \mathrm{C}$, e a do mês mais quente, superior a $22^{\circ} \mathrm{C}$, com as chuvas ocorrendo, predominantemente, no verão e no inverno, com baixas precipitações pluviais. $\mathrm{Na}$ região de $R D$, com altitudes inferiores a $400 \mathrm{~m}$, predomina o tipo Aw, tropical, com verão chuvoso e inverno seco de maio a setembro. Na região serrana de CO, o clima é do tipo Cwb, mesotérmico de inverno seco e verão ameno, com temperaturas abaixo de $22{ }^{\circ} \mathrm{C}$ (CENIBRA, 2001).

Na estimativa do estoque de $\mathrm{C}$ na biomassa (ECB) foram utilizados dados disponíveis de produtividade dos componentes de árvores de Eucalyptus spp (folhas, galhos, lenho, casca e raízes), de povoamentos implantados no espaçamento $3 \times 2 \mathrm{~m}$, com idades variando de 12 a 120 meses, e da manta orgânica depositada no solo. Os dados foram estratificados por idade e região, em intervalos de 12 meses, contados
Tabela 1 - Número de dados (n) disponíveis de biomassa dos componentes das árvores, por região.

Table 1 - Number of available data ( $n$ ) of the tree component biomass per region.

\begin{tabular}{cccccc}
\hline Componentes & \multicolumn{5}{c}{ Região $^{1}$} \\
\cline { 2 - 6 } & CO & RD & SA & SB & VI \\
\hline Folha & - & 77 & 87 & 247 & - \\
Galho & - & 44 & 118 & 244 & - \\
Lenho & - & 42 & 96 & 207 & - \\
Casca & - & 45 & 100 & 213 & - \\
Tronco & 44 & 25 & 111 & 66 & 58 \\
\hline
\end{tabular}

(1) $\mathrm{CO}=$ Cocais; $\mathrm{RD}=$ Rio Doce; $\mathrm{SA}$ = Sabinópolis; $\mathrm{SB}=$ Santa Bárbara; e VI = Virginópolis.

a partir da menor para a de maior idade. Dada a inexistência de dados de biomassa de certos componentes das árvores (Tabela 1), para algumas idades, bem como da biomassa de raízes, esses foram estimados por meio de equações de regressões, geradas no programa CurveExpert 1.3 (HYAMS, 1997), a partir dos dados existentes.

Para manta orgânica e raízes, devido ao número limitado de dados de biomassa disponíveis, nas cinco regiões (manta orgânica $=29$ e raízes $=27$ ), optouse por gerar uma equação geral para esses componentes para a região Centro-Leste de Minas. Quando da existência de dados, foram geradas equações por componente e região.

Na estimativa da biomassa dos componentes das árvores, considerou-se a relação entre esses componentes e a biomassa do tronco, obtida pelo método destrutivo, por apresentar bom grau de confiabilidade dos valores gerados em relação à biomassa real (NEVES, 2000; LOZA-BALBUENA, 2001). Dessa forma, foram geradas equações de regressão para estimativa da biomassa de todos os componentes das árvores e da partição dos componentes das árvores por idade e região de estudo. Na escolha do melhor modelo de regressão, observou-se a classificação ordenada dos modelos gerados pelo programa, por meio do coeficiente de determinação $\left(R^{2}\right)$ e da significância do modelo.

A biomassa dos componentes das árvores foi convertida em estoque de $\mathrm{C}$, multiplicando-se a biomassa pelo teor médio de $\mathrm{C}$ em cada componente da árvore, obtido por Neves (2000) para plantações de Eucalyptus grandis x urophylla na região litorânea do Estado do Espírito Santo. Esses teores médios de C foram: 524,0;

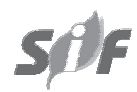

Revista Árvore, Viçosa-MG, v.35, n.4, p.895-905, 2011 
479,6; 525,1; 506,6; e 507,0 g kg-1 , respectivamente, para folhas, casca, galhos, lenho e raiz grossa. Considerando que a manta orgânica é constituída basicamente por folhas, galhos e casca, estimou-se o teor médio de C desses três componentes em 509,6 $\mathrm{g} \mathrm{kg}^{-1}$, para cálculo do estoque de C.

O estoque de carbono na biomassa (ECB) das árvores (parte aérea + raízes) e da manta orgânica, por hectare, foi obtido utilizando-se a seguinte fórmula:

$$
\begin{gathered}
\mathrm{ECB}=\mathrm{Bfs}(\mathrm{f})+\operatorname{Bgs}(\mathrm{f})+\operatorname{Bls}(\mathrm{f})+\operatorname{Bcs}(\mathrm{f})+\operatorname{Brs}(\mathrm{f}) \\
+\operatorname{Bmos}(\mathrm{f})
\end{gathered}
$$

em que ECB = estoque de carbono na biomassa $\left(\mathrm{t} \mathrm{ha}^{-1}\right)$; Bfs = biomassa de folhas seca $\left(\mathrm{t} \mathrm{ha}^{-1}\right)$; $\mathrm{Bgs}=$ biomassa seca de galhos $\left(\mathrm{t} \mathrm{ha}^{-1}\right)$; Bls = biomassa seca de lenho $\left(\mathrm{t} \mathrm{ha}^{-1}\right) ;$ Bcs = biomassa de casca seca $\left(\mathrm{t} \mathrm{ha}^{-1}\right)$; Brs = biomassa seca de raízes $\left(\mathrm{t} \mathrm{ha}^{-1}\right)$; $\mathrm{Bmos}=$ biomassa de manta orgânica seca $\left(\mathrm{t} \mathrm{ha}^{-1}\right)$; e f $=$ fator de proporção de carbono no componente.

\section{RESULTADOS E DISCUSSÃO}

As equações de regressão geradas para estimativa da biomassa dos componentes das árvores e da manta orgânica de plantações de eucalipto encontram-se na Tabela 2.

Nas regiões de CO e VI, em razão do número limitado de dados disponíveis de biomassa dos componentes das árvores e por serem dados coletados aos 96 a 120 e 85 a 120 meses de idade, respectivamente, exceto na biomassa do tronco, não foram geradas equações de regressão, sendo considerada a partição dos componentes em relação à biomassa do tronco registrada nas respectivas idades (Tabelas 2 e 3 ).

A partição da biomassa dos componentes das árvores, em função da biomassa do tronco, foi semelhante entre si em todas as idades e regiões (Tabela 3). O lenho apresenta maior contribuição para a biomassa, com média de 0,70 , seguido por raízes $(0,15)$, casca $(0,08)$, galhos $(0,04)$ e folhas $(0,02)$.

A biomassa do tronco (lenho+casca) aos 84 meses, idade usualmente utilizada na definição da data de corte da floresta nas regiões em estudo, variou de 115,83 a 168,26 t ha ${ }^{-1}$ (Tabela 4). Esses valores corresponderam, em média, a 71,9\% da produção total de biomassa. O lenho, componente de maior interesse comercial, corresponde, em média, a 64,7\% da biomassa total. Após o lenho, o sistema radicular é o componente com maior produção de biomassa, com média de 26,36 t ha ${ }^{-1}$, representando 13,5\% da biomassa total, seguido de manta orgânica, com 19,03 tha ${ }^{-1}$ (9,7\%); casca, com 14,32 tha $^{-1}$ (7,3\%); galhos, com 6,46 tha-1 (3,3\%); e folhas, com 2,99 $\mathrm{t} \mathrm{ha}^{-1}(1,5 \%)$. Esses valores se aproximam daqueles obtidos em plantios de eucalipto por Neves (2000) e Schumacker e Witschoreck (2004), com idades de 9 e 6 anos, respectivamente.

Tabela 2 - Equações de regressão para estimativa da biomassa do tronco, partição dos componentes das árvores (lenho, casca, galhos e folhas) em função da idade, por região, equações gerais para estimativa da biomassa do tronco, raízes, manta orgânica e partição dos componentes na região Centro-Leste de Minas Gerais.

Table 2 - Regression equations to estimate trunk biomass, partition of tree components (wood, bark, branches and leaves) in function of age per region and general equations to estimate biomass of trunk, roots, litter layer and partition

\begin{tabular}{|c|c|c|}
\hline Componente & Equação de regressão & $\mathbf{R}^{2}$ \\
\hline \multicolumn{3}{|c|}{ Cocais } \\
\hline Tronco & $\hat{\mathrm{Y}}_{\mathrm{T}_{\mathrm{r}}}=-4,3921+1,1143 \mathrm{i}+0,0044 \mathrm{i}^{2}$ & 0,891 \\
\hline Lenho & $\frac{W_{L}}{W_{T}}=0,90$ & - \\
\hline Casca & $\frac{W_{C}}{W_{T r}}=0,10$ & - \\
\hline Galhos & $\frac{W_{G}}{W_{T}}=0,04$ & - \\
\hline Folhas & $\frac{W_{F}^{I r}}{W_{T r}}=0,02$ & - \\
\hline
\end{tabular}
of tree components for the Central-East region of Minas Gerais.

Revista Árvore, Viçosa-MG, v.35, n.4., p.895-905, 2011 
Tabela 2 - Cont Table 2 - Cont.

Rio Doce

\begin{tabular}{|c|c|c|}
\hline Tronco & $\hat{\mathrm{Y}}_{T r}=150,9416 /(1+21,1017 \exp (-0,0925 i))$ & 0,984 \\
\hline Lenho & $\frac{W_{L}}{W_{T r}}=0,9116 /(1+0,1831 \exp (-0,0764 i))$ & 0,876 \\
\hline Casca & $\frac{W_{C}}{W_{T r}}=0,0893 /(1-1,9056 \exp (-0,0882 i))$ & 0,895 \\
\hline Galhos & $\frac{W_{G}}{W_{T r}}=0,0618 /(1-5,4656 \exp (-0,1069 i))$ & , 8 \\
\hline Folhas & $\frac{W_{F}}{W_{T r}}=0,0199 /(1-1,8713 \exp (-0,0390 \mathrm{i}))$ & 832 \\
\hline \multicolumn{3}{|c|}{ Sabinópolis } \\
\hline Tronco & $\hat{\mathrm{Y}}_{T r}=279,5691 /(1+17,4880 \exp (-0,0390 i))$ & 0,962 \\
\hline Lenho & $\frac{W_{L}}{W_{T_{r}}}=0,8902 /(1+0,8659 \exp (-0,1559 i))$ & 0,867 \\
\hline Casca & $\frac{W_{C}}{W_{T r}}=0,1101 /(1-1,4709 \exp (-0,0922 i))$ & ,880 \\
\hline Galhos & $\frac{W_{G}}{W_{T r}}=0,0185 /(1-1,0825 \exp (-0,0079 i))$ & ,9 \\
\hline Folhas & $\frac{W_{F}}{W_{T r}}=0,0187 /(1-1,8467 \exp (-0,0316 i))$ & 0,976 \\
\hline
\end{tabular}

\section{Santa Bárbara}

Tronco

Lenho

Casca

Galhos

Folhas

Tronco

Lenho

Casca

Galhos

Folhas

$$
\hat{\mathrm{Y}}_{T r}=197,147 /(1+28,1900 \exp (-0,0537 i))
$$

$$
\frac{W_{L}}{W_{T .}}=0,9000 /(1+0,3528 \exp (-0,0946 i))
$$$$
\frac{W_{C}}{W_{T^{*}}}=0,1001 /(1-2,8721 \exp (-0,1000 i))
$$$$
\frac{W_{G}}{W_{T .}}=0,0286 /(1-1,2611 \exp (-0,0156 i))
$$$$
\frac{W_{F}}{W_{T r}}=0,0063 /(1-1,0795 \exp (-0,0048 i))
$$

\section{Virginópolis}

$\hat{\mathrm{Y}}_{T r}=-3,3456+0,7123 i+0,0084 i^{2}$

$$
\begin{aligned}
& \frac{W_{L}}{W_{T r}}=0,89 \\
& \frac{W_{C}}{W_{T r}}=0,11 \\
& \frac{W_{G}}{W_{T r}}=0,04 \\
& \frac{W_{F}}{W_{T r}}=0,02
\end{aligned}
$$

0,876

0,878 
Tabela 2 - Cont

Table 2 - Cont.

Equação geral

\begin{tabular}{cl} 
Tronco & $\hat{\mathrm{Y}}_{T r}=0,5860+1,5534 i+0,0014 i^{2}$ \\
Lenho & $\frac{W_{L}}{W_{T r}}=-1,2149+0,9190 i-7,9195 \exp \left(-005 i^{2}\right)$ \\
Casca & $\frac{W_{C}}{W_{T r}}=1,1971+0,0801 i+8,5275 \exp \left(-05 i^{2}\right)$ \\
Folhas & $\frac{W_{F}}{W_{T r}}=1,7158 i^{0,1373}$ \\
Galhos & $\frac{W_{G}}{W_{T r}}=0,5264 i^{0,5029}$ \\
Raízes & $\hat{\mathrm{Y}}_{R}=176,4035\left(1-\exp \left(-0,0011 \hat{\mathrm{Y}}_{T r}\right)\right)$ \\
Manta orgânica & $\hat{\mathrm{Y}}_{M o}=3,5154+0,1943 \hat{\mathrm{Y}}_{T r}-0,0006 \hat{\mathrm{Y}}_{T r}{ }^{2}$ \\
\hline
\end{tabular}

$\hat{\mathrm{Y}}_{\mathrm{Tr}}=$ biomassa do tronco, em t/ha; $i$ = idade, em meses; $\mathrm{W}=$ biomassa; $\mathrm{L}=$ lenho; $\mathrm{Tr}=$ tronco; $\mathrm{C}=$ casca; $\mathrm{F}=$ folhas;

$\mathrm{G}=$ galhos; $\hat{\mathrm{Y}}_{\mathrm{R}}=$ biomassa de raízes; $\hat{\mathrm{Y}}_{\mathrm{Mo}}=$ biomassa de manta orgânica; e $\mathrm{R}^{2}$ = coeficiente de determinação.

Tabela 3 - Partição da biomassa dos componentes das árvores (lenho, casca, folhas, galhos e raízes), por região, em função da biomassa do tronco, numa sequência de idade.

Table 3 - Partition of the biomass of the tree components (wood, bark, leaves, branches and roots) per region, in function of trunk biomass at age sequence.

\begin{tabular}{|c|c|c|c|c|c|c|c|c|c|}
\hline \multirow[t]{2}{*}{ Componente } & \multicolumn{9}{|c|}{ Idade (mês) } \\
\hline & 24 & 36 & 48 & 60 & 72 & 84 & 96 & 108 & 120 \\
\hline \multicolumn{10}{|c|}{ Cocais } \\
\hline Lenho & 0,71 & 0,72 & 0,72 & 0,72 & 0,72 & 0,72 & 0,72 & 0,72 & 0,72 \\
\hline Casca & 0,08 & 0,08 & 0,08 & 0,08 & 0,08 & 0,08 & 0,08 & 0,08 & 0,08 \\
\hline Folhas & 0,02 & 0,02 & 0,02 & 0,02 & 0,02 & 0,02 & 0,02 & 0,02 & 0,02 \\
\hline Galhos & 0,03 & 0,03 & 0,03 & 0,03 & 0,03 & 0,03 & 0,03 & 0,03 & 0,03 \\
\hline Raízes & 0,16 & 0,16 & 0,16 & 0,15 & 0,15 & 0,15 & 0,15 & 0,15 & 0,15 \\
\hline \multicolumn{10}{|c|}{ Rio Doce } \\
\hline Lenho & 0,64 & 0,69 & 0,71 & 0,71 & 0,72 & 0,72 & 0,72 & 0,72 & 0,72 \\
\hline Casca & 0,08 & 0,08 & 0,07 & 0,07 & 0,07 & 0,07 & 0,07 & 0,07 & 0,07 \\
\hline Folhas & 0,05 & 0,03 & 0,02 & 0,02 & 0,02 & 0,02 & 0,02 & 0,02 & 0,02 \\
\hline Galhos & 0,08 & 0,05 & 0,05 & 0,05 & 0,05 & 0,05 & 0,05 & 0,05 & 0,05 \\
\hline Raízes & 0,14 & 0,15 & 0,15 & 0,15 & 0,15 & 0,15 & 0,15 & 0,15 & 0,15 \\
\hline \multicolumn{10}{|c|}{ Sabinópolis } \\
\hline Lenho & 0,58 & 0,66 & 0,69 & 0,70 & 0,71 & 0,71 & 0,72 & 0,72 & 0,72 \\
\hline Casca & 0,08 & 0,08 & 0,08 & 0,09 & 0,09 & 0,09 & 0,09 & 0,09 & 0,09 \\
\hline Folhas & 0,09 & 0,03 & 0,02 & 0,02 & 0,02 & 0,02 & 0,02 & 0,02 & 0,02 \\
\hline Galhos & 0,12 & 0,07 & 0,06 & 0,04 & 0,04 & 0,03 & 0,03 & 0,03 & 0,03 \\
\hline Raízes & 0,13 & 0,15 & 0,15 & 0,15 & 0,15 & 0,15 & 0,15 & 0,15 & 0,14 \\
\hline \multicolumn{10}{|c|}{ Santa Bárbara } \\
\hline Lenho & 0,55 & 0,65 & 0,68 & 0,70 & 0,71 & 0,72 & 0,72 & 0,73 & 0,73 \\
\hline Casca & 0,08 & 0,08 & 0,08 & 0,08 & 0,08 & 0,08 & 0,08 & 0,08 & 0,08 \\
\hline Folhas & 0,10 & 0,05 & 0,03 & 0,03 & 0,02 & 0,02 & 0,02 & 0,01 & 0,01 \\
\hline Galhos & 0,14 & 0,07 & 0,05 & 0,04 & 0,04 & 0,03 & 0,03 & 0,03 & 0,03 \\
\hline Raízes & 0,13 & 0,14 & 0,15 & 0,15 & 0,15 & 0,15 & 0,15 & 0,15 & 0,15 \\
\hline \multicolumn{10}{|c|}{ Virginópolis } \\
\hline Lenho & 0,71 & 0,71 & 0,71 & 0,71 & 0,71 & 0,71 & 0,71 & 0,72 & 0,72 \\
\hline Casca & 0,09 & 0,09 & 0,09 & 0,09 & 0,09 & 0,09 & 0,09 & 0,09 & 0,09 \\
\hline Folhas & 0,02 & 0,02 & 0,02 & 0,02 & 0,02 & 0,02 & 0,02 & 0,02 & 0,02 \\
\hline Galhos & 0,03 & 0,03 & 0,03 & 0,03 & 0,03 & 0,03 & 0,03 & 0,03 & 0,03 \\
\hline Raízes & 0,16 & 0,16 & 0,16 & 0,16 & 0,15 & 0,15 & 0,15 & 0,15 & 0,15 \\
\hline
\end{tabular}

Revista Árvore, Viçosa-MG, v.35, n.4., p.895-905, 2011 


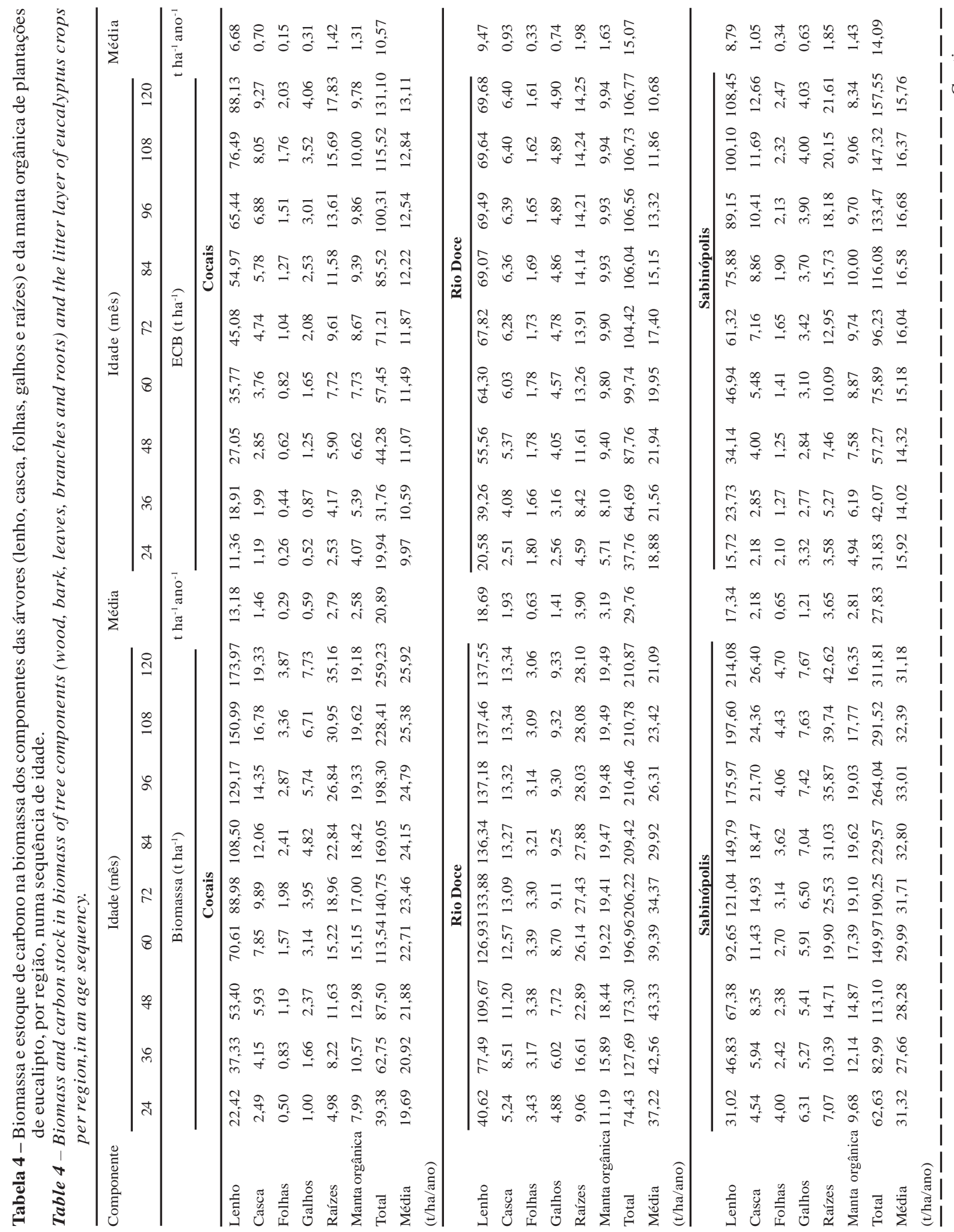




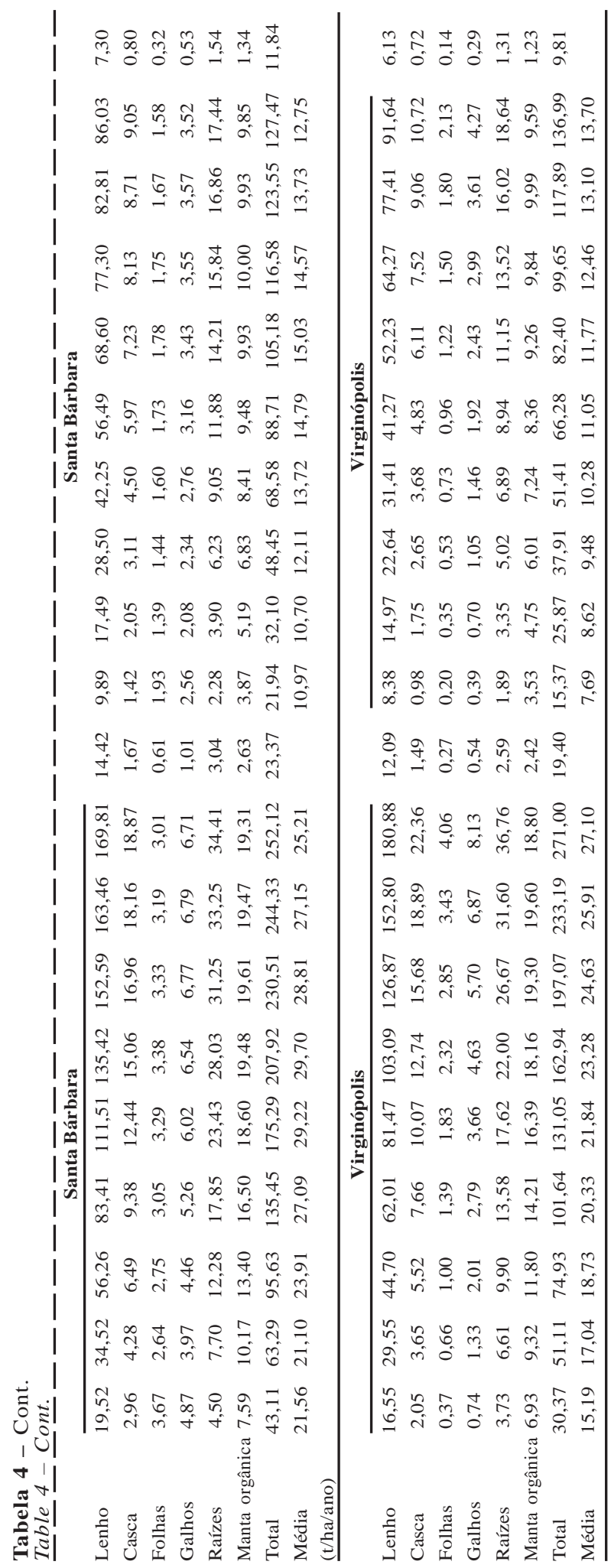

A região de SA apresentou maior produtividade (32,80 tha $\left.^{-1}\right)$ aos 84 meses de idade e, consequentemente, maior estoque de C. A partir dos 84 meses, o incremento médio em biomassa tende a se estabilizar nas regiões de RD e SB e, aos 96 meses de idade, na região de SA. Contudo, nas regiões de CO e VI a produtividade e a alocação de $C$ continuam crescentes, indicando que o incremento médio anual não se estabilizou. Essa condição indica que a idade de corte das plantações florestais, nessas duas regiões, dos pontos de vista biológico e econômico, deve ser superior a sete anos (Tabela 4).

A produção de biomassa do sistema radicular variou de 22,0 a 31,03 tha-1, aos 84 meses de idade, nas cinco regiões, correspondendo a 13,5 e $20,9 \%$ da biomassa total e do lenho, respectivamente (Tabela 4). Esses valores se aproximam daqueles encontrados por Mello et al. (1998) e Santana (2000), ao avaliarem a produção de biomassa da parte aérea e do sistema radicular de povoamentos de E. grandis, aos 7 anos de idade. Esses autores verificaram que a biomassa radicular correspondeu, respectivamente, a $18,0 \%$ e $19,5 \%$ da biomassa total e do lenho.

A produção de biomassa da parte aérea e das raízes é influenciada pela capacidade produtiva do sítio e condições climáticas (MOKANY et al., 2005; CASTILHO et al., 2006; WANG et al., 2008). Tem sido registrada maior produção de biomassa de raízes em sítios onde os estresses nutricional e, ou, hídrico são mais acentuados(GONÇALVES, 1994; MELLO; GONÇALVES, 2008). Quando a disponibilidade de água e nutrientes é limitada, as árvores tendem a alocar, em termos relativos, mais fotoassimilados nas raízes, em detrimento dos demais componentes da árvore (GONÇALVES; MELLO, 2000). Tal tendência foi constatada neste trabalho, sendo a maior produção de biomassa radicular encontrada nas regiões RD (27,9 t ha-1), SA (31,0 t ha-1) e SB (28,0 t ha-1), respectivamente, com déficit hídrico de 300,203 e $195 \mathrm{~mm}^{2} \mathrm{nno}^{-1}$.

Independentemente da região, constatou-se aumento do estoque de $\mathrm{C}$ orgânico nos componentes de árvores de eucalipto com o aumento da idade, exceto no componente folhas, nas regiões de RD e SB. Entretanto, a biomassa e o estoque de C orgânico na manta orgânica tenderam a decrescer em todas as regiões e em idades mais avançadas. 
Entre todos os componentes analisados, os maiores estoques de $\mathrm{C}$ foram registrados no lenho e nas raízes, em virtude da maior biomassa produzida. A região de SA, considerada neste trabalho como o sítio de melhor qualidade, foi a mais produtiva entre todas, com 75,9 e 15,7 t ha-1, respectivamente, de C estocado no lenho e nas raízes, aos 84 meses de idade (Tabela 4). Os resultados de lenho aproximam-se dos apresentados por Soares et al. (2005) ao estimarem o estoque de C no lenho, em povoamentos de E. grandis, aos 80 meses de idade, que encontraram valores de $\mathrm{C}$ estocado variando de 43,9 a 82,3 t ha-1, nos sítios de pior e melhor qualidade, respectivamente. Também no lenho, aos 84 meses, Silva et al. (2008) encontraram, em plantios de $E$. grandis, estoque de $\mathrm{C}$ em sítios de pior e melhor qualidade variando de 40,2 a 97,7 t ha-1 . Considerandose a idade de 72 meses, o estoque de C no lenho, nas cinco regiões avaliadas, foi, em média, 54,4 t ha${ }^{1}$, valor superior ao encontrado por Paixão et al. (2006), que estimaram em 39,0 tha $^{-1}$ o estoque de $\mathrm{C}$ em plantios de E. grandis, nessa idade, na região de Viçosa, MG. Com relação ao estoque de $\mathrm{C}$ nas raízes, o valor médio foi de 11,49 $\mathrm{t} \mathrm{ha}^{-1}$, superior ao estimado por Schumacher e Witschoreck (2004), que encontraram 8,89 t ha-1 de C estocado em plantios de eucalipto também com 72 meses de idade.

\section{CONCLUSÕES}

A produtividade média e o estoque de carbono na biomassa, por região, aos 120 meses de idade apresentaram a seguinte sequência: Sabinópolis > Virginópolis > Cocais > Santa Bárbara > Rio Doce.

O lenho contribuiu, em média, com 126,63 tha-1 (64,7\%) da biomassa total, seguido pelo sistema radicular, com 26,36 t ha-1 (13,5\%), manta orgânica, com 19,03 t ha-1 (9,7\%); casca, com 14,32 t ha-1 (7,3\%); galhos, com 6,46 tha-1 (3,3\%); e folhas, com 2,99 $\mathrm{t} \mathrm{ha}^{-1}$ (1,5\%).

Maior estoque de carbono foi registrado em Sabinópolis, aos 84 meses de idade, com média de $16,58 \mathrm{t} \mathrm{ha}^{-1} \mathrm{ano}^{-1}$, seguido por Rio Doce com 15,16 t ha ${ }^{-1}$ ano $^{-1}$, Santa Bárbara com 15,04 t ha-1 ano ${ }^{-1}$, Cocais com 12,23 t ha ${ }^{-1}$ ano $^{-1}$ e Virginópolis com 11,77 t ha $^{-1}$ ano $^{-1}$.

O estoque médio de carbono na biomassa de plantações de eucalipto, aos 84 meses de idade, na região CentroLeste de Minas Gerais foi de 14,15 t ha ${ }^{-1} \mathrm{ano}^{-1}$.

\section{REFERÊNCIAS}

ALVAREZ, E. Composición florística diversidad, estructura y biomasa de un bosque inundable em la Amazonia colombiana. 1993. 143f. Tesis (Mestrado em Biologia) - Universidad de Antioquia, 1993.

BASHKIN, M. A.; BINKLEY, D. Changes in soil carbon following afforestation in Hawaii. Ecology, v.79, n.3, p.828-833, 1998.

BEETS, P. K. et al. Description and validation of C-change: a model for simulating carbon content in managed Pinus radiata stands. New Zealand Journal of Forestry Science, v.29, n.3, p.409-427, 1999.

BRASIL. Ministério da Ciência e Tecnologia MCT. Estimativa da quantidade de carbono fixado pelas florestas plantadas. 2001. (Disponível na internet <www.mct.gov.br/clima>).

BROWN, S.; LUGO, A. E.; CHAPMAN, J. Biomass of tropical tree plantations and implications for the global carbon budget. Canadian Journal of Forest Research, v.16, n.2, p.390-394, 1986.

CASTILHO, C. V. D. et al. Variation in aboveground tree live biomass in a central Amazonian forest: effects of soil and topography. Forest Ecology and Management, v.234, n.1/3, p.85-96, 2006.

CELULOSE NIPO-BRASILEIRA S.A. - CENIBRA. Relatório do Levantamento Semidetalhado de Solos da Cenibra: Fase 2. Belo Oriente: Cenibra, 2001. v.1. 101p.

DELANEY, M. et al. The distribution of organic carbon in major components of Forests located in five life zones of Venezuela. Journal of Tropical Ecology, v.13, n.5, p.697-708, 1997.

DEWAR, R. C.; CANNELL, M. G. R. Carbon sequestration in the tree, products and soils of forest plantations: an analysis using UK examples. Tree Physiology, v.11, n. 1, p.49-72, 1992.

DIAZ-BALTEIRO, L.; RODRIGUEZ, L. C. E. Optimal rotation on Eucalyptus plantation including carbon sequestration - a comparison of results in Brazil and Spain. Forest Ecology Management, v.229, n.1/3, p.247-258, 2006. 
FERREIRA, M. G. M. An analysis of the future productivity of Eucalyptus grandis plantations in the "cerrado" region in Brasil: A nutrient cycling approach. 1984. 230f. Thesis (Ph.D.) - University of British Columbia, Vancouver, 1984.

FLINT, E. P.; RICHARDS, J. F. Trends in carbon content of vegetation in South and Southeast Asia associated with changes in land use. In: DALE, V. H. (Ed.) Effects of land-use changes on atmospheric concentrations. South and Southeast Asia as a case study. New York: Springer-Verlag, 1994. p.201-299.

GIFFORD, R. Carbon content of aboveground tissues of forest and woodland trees. National Carbon Accouting System Technical Report $n^{\circ} 22$. Australian Greenhouse Office, 2000. 27p.

GONÇALVES, J. L. M. Características do sistema radicular de absorção do Eucalyptus grandis sob diferentes condições edáficas. 1994. 84f. Tese (Livre Docência) - Escola Superior de Agricultura Luiz de Queiroz, Piracicaba, 1994.

GONÇALVES, J. L. M.; MELLO, S. L. M. O sistema radicular das árvores. In: GONÇALVES, J. L. M.; BENEDETTI, V. (Ed.) Nutrição e fertilização florestal. Piracicaba, IPEF, 2000. p.221-267.

HOEN, H.; SOLBERG, B. Potential and economic efficiency of carbon sequestration in forest biomass through silvicultural management.

Forest Science, v.3, n.2, p.429-451, 1994.

HYAMS, D. G. CurveExpert 1.3: A comprehensive curve fitting system for Windows. http://curveexpert.webhop.biz/. Starkville: 1997. [software].

\section{INTERGOVERNMENTALPANELONCLIMATE}

CHANGE - IPCC. Land use change and forestry. In: Revised 1996 guidelines for national GHG inventories: reference manual. 1996. p. 5.1-5.75.

LELES, P.S.S.; NETO, S.N.O.; SILVA, E. Captura e fluxo de $\mathrm{CO}_{2}$ atmosférico pelas florestas plantadas e manejadas. Boletim Técnico SIF, v.7, p.1-13, 1994.
LITTON, C.; RAICH, J. W.; RYAN, M. G. Carbon allocation in forest ecosystems. Global Change Biology, v.13, n.10, p.2089-2109, 2007.

LOZA-BALBUENA, I. Impacts carbon trading in afforestation projects in Uruguay. 2001. 121f. Tesis (Mestrado em Ciência Ambiental) - University of Canterbury, New Zealand, 2001.

MARCOLIN, M. Primeiro inventário brasileiro de emissões antrópicas de gases de efeito estufa: Emissões e remoções de dióxido de carbono por mudanças nos estoques de florestas plantadas. Brasília: Ministério da Ciência e Tecnologia, 2002. 47p.

MELLO, S. L. M.; GONÇALVES, J. L. M. Equações para estimar a biomassa da parte aérea e do sistema radicular em povoamentos de Eucalyptus grandis em sítios com produtividades distintas. Revista Árvore, v.32, n.1, p.101-111, 2008.

MELLO, S. L. M.; GONÇALVES, J. L. M.; OLIVEIRA, L. E. G. Características do sistema radicular em povoamentos de eucaliptos propagados por sementes e estacas. Scientia Forestalis, v.54, n.1, p.16-26, 1998.

BRASIL, MINISTÉRIO DA CIÊNCIA E TECNOLOGIA - MCT. Estimativa da quantidade de carbono fixado pelas florestas plantadas. 2001. (Disponível na internet $<$ www.mct.gov.br/clima $>$ ).

MOKANY, K.; RAISON, R. J.; PROKUSHKIN, A. S. Critical analysis of root: shoot ratios in terrestrial biomes. Global Change Biology, v.11, n.1, p.1-13, 2005.

MONTAGU, K. D. et al. Developing general allometric relationship for regional estimates of carbon sequestration - an example using Eucalyptus pilularis from seven contrasting sites. Forest Ecology Management, v.204, n.1, p.113-127, 2005.

NEVES. J. C. L. Produção e partição de biomassa, aspectos nutricionais e hídricos em plantios clonais de eucalipto na região litorânea do Espírito Santo. 2000. 191f. Tese (Doutorado em Produção vegetal) - Universidade Estadual Norte Fluminense, Campos dos Goytacazes, 2000. 
ORTIZ, R. Costa Rican secondary forest: an economic option for joint implementation initiatives to reduce atmospheric $\mathbf{C O}_{2}$. Draft paper presented for inclusion in the Beijer Seminar in Punta Leona. Costa Rica: 1997. 19p.

PAIXÃO, F. A. et al. Quantificação do estoque de carbono e avaliação econômica de diferentes alternativas de manejo em um plantio de eucalipto. Revista Árvore, v.30, n.3, p.411-420, 2006.

SALES, M. H. et al. Improving spatial distribution estimation of forest biomass with geostatistics:a case study for Rondonia, Brazil. Ecological Modelling, v.205, n. 1/2, p.221-230, 2007.

SANTANA, R. C. Predição de biomassa e alocação de nutrientes em povoamentos de eucalipto no Brasil. 2000. 59f. Tese (Doutorado em Solos e Nutrição de Plantas) Universidade Federal de Viçosa, Viçosa, MG, 2000.

SCHROEDER, P. E. Carbon storage potencial of short rotation tropical tree plantations. Forest Ecology Management, v.50, n.1/2, p.31-41, 1992.

SCHUMACHER, M. V.; WITSCHORECK, R. Inventário de carbono em povoamentos de Eucalypus spp nas propriedades fumageiras do sul do Brasil: "Um estudo de caso". In: SANQUETTA, C. R.; BALBINOT, R.; ZILIOTTO, M. A. B., (Ed.)

Fixação de carbono: atualidades, projetos e pesquisas. Curitiba: AM Impressos, 2004. p.111-124.
SILVA, R. F. et al. Projeção do estoque de carbono e análise da geração de créditos em povoamentos de eucalipto. Revista Árvore, v.32, n.6, p.979-992, 2008.

SOARES, C. P. B.; LEITE, H. G.; GÖRGENS, E. B. Equações para estimar o estoque de carbono no fuste de árvores individuais e em plantios comerciais de eucalipto. Revista Árvore, v.29, n.5, p.711-718, 2005.

STAPE, J. L.; BINKLEY, D.; RYAN, M. G. Production and carbon allocation in a clonal Eucalyptus plantation with water and nutrient manipulations. Forest Ecology Management, v.255, n.3/4, p.920-930, 2008.

VÉLEZ, V. H. G.; ARANGO, G. J. L. Metodología para la cuantificación de existencias y flujo de carbono en plantaciones forestales. In: SIMPOSIO INTERNACIONAL MEDICIÓN Y MONITOREO DEL LA CAPTURA DE CARBONO EN ECOSISTEMAS FORESTALES. Valdivia: 2001. $18 p$.

WANG, X.; FANG, J.; ZHU, B. Forest biomass and root-shoot allocation in northeast China. Forest Ecology and Management, v.255, n.12, p.4007-4020, 2008. 
